\title{
El practicum de magisterio a examen: reflexiones de un grupo de estudiantes de la Universidad de Cantabria
}

\author{
Ángela Saiz-Linares y Noelia Ceballos-López
}

\section{RESUMEN}

Este trabajo tiene como objetivo comprender y valorar la experiencia de practicum de maestros desde la perspectiva de los alumnos de la Universidad de Cantabria (España) y avanzar algunos elementos para la mejora. Se ha utilizado una metodología cualitativa y se han analizado informaciones procedentes de entrevistas en profundidad realizadas a una muestra compuesta por seis participantes. Los resultados evidencian una valorización del practicum sobre la formación de carácter más teórico, en unas reflexiones donde sobresale una visión tecnificada de la enseñanza. Asimismo, se muestra cierta insatisfacción con la supervisión del tutor universitario y el portfolio como herramienta de reflexión. Discutimos, finalmente, sobre el papel del tutor del centro educativo y la necesidad de conformar espacios de reflexión compartida.

Palabras clave: practicum, tutorización, portfolio, formación reflexiva, España.

Ángela Saiz-Linares

saizla@unican.es Española. Doctora en Educación por la Universidad de Cantabria, España. Profesora en el Departamento de Educación de la Universidad de Cantabria, España. Temas de investigación: formación inicial de los docentes y el practicum, dinámicas de inclusión y exclusión educativa y social, y la voz del alumnado. de Educación de la Universidad de Cantabria, España, y miembro del equipo de investigación IN-PARES, como docente e investigadora. Temas de investigación: inclusión educativa, procesos de participación y voz del alumnado, investigación participativa. 


\section{0 practicum de magistério a exame: reflexões de um grupo de estudantes da Universidade de Cantábria}

\section{RESUMO}

Este trabalho tem por objetivo compreender e examinar a experiência de practicum de professores pela perspectiva dos alunos da Universidade de Cantábria (Espanha) e avançar alguns elementos para a melhora. Utilizou-se uma metodologia qualitativa e analisou-se informações procedentes de entrevistas em profundidade realizadas a uma amostra composta por seis participantes. Os resultados demonstram uma valorização do practicum sobre a formação de caráter mais teórico, em umas reflexões onde sobressai uma visão técnica do ensino. Também, percebe-se certa insatisfação com a supervisão do tutor universitário e o portfólio como ferramenta de reflexão. Discutimos, finalmente, sobre o papel do tutor do centro educativo e a necessidade de conformar espaços de reflexão comum.

Palavras chave: practicum, autorização, portfólio, formação reflexiva, Espanha.

\section{The practicum in teacher education under scrutiny: reflections of a student group enrolled at the Universidad de Cantabria}

\section{ABSTRACT}

This paper aims to understand and value the practicum experience of teachers from the perspective of the students enrolled at the University of Cantabria (Spain) and to propose some elements for improvement. A qualitative methodology has been used and information from in-depth interviews with a sample of six participants has been analyzed. The results show that the students value practicum far above more theoretical training, in some reflections where a vision of teaching based on technical support stands out. Likewise, a certain dissatisfaction is shown with the supervision of the university tutor and the portfolio as a tool for reflection. Finally, the authors discuss the role of the school tutor and the need to create spaces for shared reflection.

Key words: practicum, tutoring, portfolio, reflective training, Spain.

Recepción: 26/06/17. Aprobación: 8/12/17. 


\section{Introducción}

El contexto universitario español se ha visto inmerso recientemente en un profundo proceso de cambio hacia el Espacio Europeo de Educación Superior (EEES). En este marco de convergencia, la formación inicial del profesorado ha sufrido una importante reestructuración. La ampliación en un año de la carrera universitaria de magisterio y un aumento considerable del periodo de prácticas se perfilan como dos de los cambios más significativos. Sin embargo, sabemos que no basta con aumentar los años de educación para mejorar la calidad de la formación del profesorado (Imbernón y Colén, 2015). Algunos estudios nacionales (Aneas et al., 2013; Martínez Bonafé, 2013) apuntan que este proceso de cambio ha conllevado modificaciones formales, pero no una verdadera reflexión sobre las necesidades formativas actuales ni el cuestionamiento de algunos elementos de la cultura universitaria que dificultan una reforma más radical. Por ello, se requiere orientar el debate hacia cuestiones más profundas que afectan a la estructura, al currículum y a la cultura de las instituciones de formación de maestros (RodríguezHoyos et al., 2015).

El practicum se presenta como uno de los ámbitos fundamentales donde centrar el debate pues, aun habiéndose ampliado el número de horas de dedicación, el modelo real de prácticas aparece, en esencia, inalterado. En tal sentido, la experiencia de la primera promoción de graduados constituye una oportunidad para reflexionar sobre el modelo de practicum que tenemos y avanzar mejoras en algunos aspectos organizativos y curriculares fundamentales en este periodo.

$\mathrm{Al}$ amparo de estas necesidades desarrollamos en la Universidad de Cantabria una investigación cuyo objetivo es alcanzar un conocimiento más profundo de la experiencia formativa de prácticas desde la mirada multifocal que nos aporta la triada de agentes involucrados (alumnado, tutores de los centros educativos y supervisores de la universidad). El propósito último de este estudio exploratorio es construir un análisis multivocal del practicum que permita avanzar algunos elementos de mejora. Concretamente, presentamos una de estas aristas de análisis, aquella que trata de comprender y valorar la experiencia de practicum (aspectos estructurales, pedagógicos y organizativos) desde la percepción de los estudiantes.

\section{Marco teórico}

$\mathrm{El}$ practicum es una pieza fundamental en los procesos de formación inicial de docentes y una ocasión irremplazable para el aprendizaje de la profesión (Beck y Kosnik, 2002; González-Sanmamed y Fuentes, 2011; Sorensen, 2014). Con la última reforma universitaria hacia el EEES y su vocación profesionalizadora, el practicum de magisterio ha experimentado un reseñable crecimiento en el contexto español, convirtiéndose en la asignatura troncal con mayor número de créditos dentro del plan de estudios.

Si bien es reconocida su relevancia en la formación de nuestros futuros docentes, debemos señalar que, en el contexto español, carecemos de un modelo de practicum unificado (Zabalza, 2011). A pesar de las diferentes conceptualizaciones, existe cierto consenso al apuntar algunos rasgos esenciales, como la ubicación de este periodo en centros externos de la universidad (Ventura, 2005) o el encuentro que, bajo este marco, se produce entre el mundo formativo y el productivo (Correa, 2015). También reconocemos como habituales las interpretaciones que sitúan la relación entre la teoría y la práctica como elemento cardinal de estos programas. De hecho, González Sanmamed (2001) enuncia que el valor que otorguemos a este periodo formativo va a depender del modelo de relación teoría-práctica elegido. Wilson e I'Anson (2006) consideran que el practicum se sitúa en un espacio intermedio entre la universidad y la escuela que permite construir, a través de la reflexión, un puente para minimizar la "brecha teoría/práctica" en los programas de formación inicial del profesorado. Así, el acercamiento que genera 
entre el estudiante y los contextos reales de trabajo y la oportunidad de establecer relaciones entre los conocimientos propios de la academia y los que provienen de los escenarios profesionales parecen perfilarse como los rasgos esenciales que uniforman las diferentes conceptualizaciones.

Por otra parte, al planificar los programas de practicum, Zabalza (2011) señala la importancia de considerar algunos elementos que van a determinar su desarrollo.

Uno de estos factores condicionantes se localiza, según este autor, en el ámbito organizativo, muy convulso a nivel administrativo. Debido al elevado número de alumnos, el practicum de magisterio suele desarrollarse con bastante desorden y dependiendo de la idiosincrasia de unos centros educativos que, en general, son seleccionados con poca precisión. No obstante, conocemos que la experiencia de prácticas favorece un mejor aprendizaje si está vinculada a procesos de innovación dentro del centro (Zabalza, 2007; Zeichner, 2005). Valle y Manso (2010) identifican cuatro factores que definen a los "buenos centros de prácticas": planteamientos pedagógicos claros y compartidos por los miembros del centro; implicación y participación de las familias; estilo de gobierno basado en un liderazgo participativo; cultura de solución de problemas explicitada en planes concretos de intervención educativa.

Por otro lado, es habitual que aspectos de tipo logístico debiliten unos esfuerzos que habrían de dedicarse a cuestiones relacionadas con la calidad del proceso formativo como son los aspectos curriculares. Zeichner (2010) y Zabalza (2013) reclaman que la propuesta de practicum debe estar integrada en un plan de formación unitario y global, donde la coherencia con el resto de asignaturas del plan de estudios se antoja imprescindible.

Otro elemento a considerar es la función del supervisor de la facultad (Correa, 2015; Korthagen et al., 2006; Mena et al., 2015). En un marco de formación reflexiva que propicie la armonización de los contenidos teóricos y prácticos, el papel del supervisor se define por orientar los procesos reflexivos y proporcionar a los alumnos oportunidades de aprendizaje significativo. Cabría considerar también el tipo de instrumentos que utiliza para evaluar al estudiantado. En los últimos años, una gran parte de facultades de educación de todo el mundo están adoptando el portfolio como instrumento para este fin (Zabalza, 2011). Podríamos definir este artefacto como una carpeta de trabajo que permite recopilar información y documentar evidencias que ayudan al alumno a reflexionar sobre su práctica. En este sentido, Cebrián de la Serna (2010: 185) subraya que, además de su virtualidad para tomar decisiones y evaluar, encierra grandes posibilidades para "recopilar experiencias, reflexionar sobre las mismas y facilitar que el estudiante gestione su aprendizaje".

Asimismo, la función tutorial del docente de escuela aparece como primordial en el proceso de aprendizaje del estudiante. Sin embargo, este componente del practicum parece configurarse como especialmente problemático (Ferrier-Kerr, 2009). Zeichner $(2002,2005)$ considera que algunos de los obstáculos residen en la inexistencia de una formación específica de los maestros para este fin y de unas condiciones institucionales que faciliten su labor. La ausencia de precisión respecto a qué tienen que evaluar aparece también como otro elemento dificultoso (Smith, 2010). Para Zeichner (2002) esta falta de definición de sus funciones, sumada a la ausencia apoyos, puede dar lugar a actuaciones poco formativas, como dejar hacer sin ningún tipo de orientación al estudiante o, en el otro extremo, limitar en exceso sus actuaciones. Melek (2012) y Clarke et al. (2014) identificaron algunas de las funciones que los tutores perciben como sus responsabilidades, que van desde proveedores de feedback y modeladores de la práctica hasta agentes de socialización y promotores de la reflexión. Por otra parte, el estudio desarrollado por Patrick (2013) evidencia una tendencia de los tutores de escuela a situarse en un rol experto con respecto a 
los estudiantes y a considerar la estancia del alumnado en sus aulas como una oportunidad de asimilar la profesión docente. En cualquier caso, parece que la urgencia está ahora en especificar oficialmente unas funciones en lugar de dejar la labor de mentorazgo a merced de cada tutor.

\section{Contextualización de nuestro practicum}

En el caso de las titulaciones de maestro de la Universidad de Cantabria, contamos con tres asignaturas de practicum. El Practicum III tiene una duración de 16 semanas en el último semestre de su formación universitaria (de febrero a mayo). Constituye un modelo de formación intensivo que se complementa con cuatro periodos cortos de formación práctica discontinua (en noviembre y febrero). Esta formación más breve y extensiva en el tiempo se distribuye entre los cursos de segundo y tercero de la titulación y se corresponde con las asignaturas de Practicum I y Practicum II. En los tres periodos, los estudiantes combinan su asistencia a un centro escolar con tareas de reflexión que desarrollan en un portfolio de prácticas. Además, se contempla la realización de, al menos, dos reuniones con el supervisor universitario para orientar la elaboración del portfolio y guiar su trabajo en los centros.

\section{Marco metodológico}

Este trabajo se asienta en los pilares de la tradición cualitativa (Flick, 2009; Denzin y Lincoln, 2012), que permite comprender el fenómeno de estudio de manera profunda y situada. Investigar sobre la asignatura de practicum requiere zambullirse en las interpretaciones (Rapley, 2014) que nuestros estudiantes y egresados confieren a su experiencia de formación práctica en los diferentes años académicos y profundizar, consecuentemente, en los significados que han construido alrededor de los diferentes componentes que configuran este periodo formativo.

La muestra se compuso de seis estudiantes que trataban de representar la experiencia de practicum en los diferentes cursos de la titulación, así como en las especialidades de infantil (I) y primaria $(\mathrm{P})$ : dos egresados (P1 y P3) que habían completado las tres experiencias de practicum, dos alumnos que cursaban el último curso $\left(4^{\circ}\right)$ (I1 e I2) y que habían realizado el Practicum I y el Practicum II y, finalmente, dos alumnos de tercero (P2 e I3) que habían completado el Practicum I. La técnica de muestreo utilizada ha sido intencional basada en criterios (Colás, 1998), de entre los que destacamos las posibilidades de acceso, la representación de los diferentes cursos de la titulación, especialidades (educación infantil y primaria) y menciones y, finalmente, la posibilidad de contar con alumnos que han realizado sus prácticas en centros educativos de diferentes características (en cuanto a titularidad, localización, etcétera).

Respecto a las técnicas de producción de información (Díaz de Rada, 2007), hemos desarrollado seis entrevistas semi-estructuradas (Kvale, 2011) que han posibilitado un tratamiento cualitativo de la información, permitiendo penetrar en los significados y hacer explícitas algunas apreciaciones que los participantes en esta investigación atribuyen a su experiencia de practicum y a las actividades desarrolladas durante el mismo (Hammersley y Atkinson, 2003).

Para el análisis de los datos utilizamos un sistema de codificación temática (Angrosino, 2012) en el que definimos las categorías de análisis y los códigos (Huber, 2003). Asimismo, empleamos estrategias de tipo inductivo y deductivo: si bien partimos de un esquema inicial de variables a analizar, el trabajo desarrollado con los datos exigió redefinir algunas de esas categorías y códigos durante el proceso de análisis (Tójar, 2006). 
Tabla 1. Categorías de análisis

\begin{tabular}{|l|l|}
\hline \multicolumn{1}{|c|}{ Categorías } & \multicolumn{1}{c|}{ Definición } \\
\hline Sentido del practicum en el proceso formativo & $\begin{array}{l}\text { Reflexiones acerca de las expectativas y el valor formativo } \\
\text { que confieren al practicum }\end{array}$ \\
\hline Papel de los tutores de los centros y actividades desarrolladas & $\begin{array}{l}\text { Declaraciones sobre las actividades desarrolladas en el } \\
\text { centro de prácticas, el rol desarrollado por el tutor y las } \\
\text { estrategias de análisis sobre la práctica desplegadas }\end{array}$ \\
\hline Papel de los supervisores de la universidad & $\begin{array}{l}\text { Informaciones y reflexiones acerca del rol del supervisor } \\
\text { universitario en cuanto al seguimiento y las actividades } \\
\text { desarrolladas }\end{array}$ \\
\hline Portfolio de practicum & $\begin{array}{l}\text { Informaciones sobre el valor del portfolio como } \\
\text { herramienta de reflexión }\end{array}$ \\
\hline
\end{tabular}

\section{Resultados \\ El practicum y su sentido en el proceso de formación}

Para comprender el impacto del practicum en nuestros alumnos debemos entender, primeramente, qué sentido le otorgan en su formación como maestros. Las concepciones con que los estudiantes enfrentan este momento formativo influirán en cuestiones sustanciales como el proceso de selección de los centros, las acciones que desarrollen durante su estancia en los colegios y el enfoque con que emprendan la elaboración del portfolio.

De forma mayoritaria, los estudiantes reconocen que el practicum es un momento crucial de acercamiento a la práctica, llegando a considerar este periodo como el más valioso de la titulación: ["es aquel [con el que] cuando acabe la carrera voy a saber cómo actuar en un aula"] <entrevista(E) Il>. Sin embargo, son dos interpretaciones bien diferentes las que asoman cuando ahondamos en las relaciones entre la teoría y la práctica que asumen.

En una primera comprensión, ambos conceptos constituyen dos fenómenos independientes (González-Sanmamed, 2001). De esta suerte, los estudiantes identifican la teoría con los conocimientos ofrecidos desde la academia y la práctica con aquellos que tienen lugar en la realidad del centro de prácticas. Por esta razón, una de las cuestiones que más valoran de su estancia en los colegios es la posibilidad de acceder a la acción, de enfrentarse autónomamente a la tarea docente: pueden ensayar el rol de tutor, comprender cómo funciona un aula y, en definitiva, "aprender a hacer". "En principio consiste en elegir un centro, ir a él y hacer las funciones que llevaría a cabo un tutor" (EI2).

En consonancia con un paradigma técnico instrumental, la valorización que realizan de la acción más directa les lleva a desmerecer aquellos contenidos académicos que no son directamente aplicables, reclamando a la universidad mayores cuotas de conocimiento en estrategias y técnicas de enseñanza.

\footnotetext{
Aprendes más cuando estás con los niños delante y actúas con ellos que cuando estás en clase estudiando cosas que muchas veces no te sirven para luego llevarlo al colegio (EP1).

Nos faltan muchos conocimientos de cómo enseñar a los niños las cosas (EP3).
} 
No obstante, también encontramos interpretaciones sobre el periodo de prácticas como un espacio de aprendizaje que permite hibridar los conocimientos provenientes de ambos contextos, universidad y escuela. Esta segunda idea se vincula con el desarrollo de un "practicum curricular" y plenamente formativo, "destinado a enriquecer la formación básica complementando los aprendizajes académicos (teóricos y prácticos) con la experiencia (también formativa) en centros de trabajo" (Zabalza, 2006: 314). "Las prácticas son el puente entre lo que veamos teóricamente y lo que vamos a hacer en un aula" (EIl).

Bajo esta significación, la formación recibida en la universidad aportaría un acervo pedagógico y fomentaría el desarrollo de algunas claves con las cuales comprender la práctica pedagógica. El valor de este tipo de aprendizajes, lejos del utilitarismo propio del paradigma tecnocrático, tiene más que ver con la posibilidad de imaginar otras formas de actuación y organización posibles.

A pesar de que esta comprensión está presente en algunos discursos, la tendencia a divorciar los constructos de teoría y práctica sobresale con fuerza en casi todos los participantes en algún momento de la entrevista. Esta realidad nos interpela a replantear la práctica universitaria y a tratar de dar respuesta a la siguiente cuestión: ¿por qué nuestros alumnos desarrollan una visión tecnocrática de la educación?

En primer lugar, estas concepciones reduccionistas entre teoría y práctica, entre formación académica y practicum, pueden explicarse por una organización del plan de estudios exageradamente fragmentada en disciplinas. Por otro lado, existe una tendencia a trabajar desde una perspectiva excesivamente teórica y descontextualizada en algunas asignaturas universitarias, desligando dichas teorías de las experiencias concretas donde las mismas toman sentido. "Algunas cosas son muy utópicas (de las que aprendes en la universidad) [...] falta relación entre la teoría y la práctica" (EI3).

Ello nos impele a plantear nuestras aulas universitarias como lugares en los que construir la teoría educativa a partir de casos prácticos o experiencias docentes concretas, al tiempo que a definir formas de hacer, ser y estar en las aulas escolares que se desprendan de los conocimientos y reflexiones de orientación más teórica. Este parece el único modo factible de establecer puentes entre los conocimientos pedagógicos propios de la academia y lo que ocurre en la realidad de aula a la que en sus prácticas y en su futura docencia se van a enfrentar. "Hay cosas que sí te tienen que enseñar. Que luego cuando tú vayas al aula con unos alumnos concretos decides [...] cosas que son muy básicas en colegio, que yo creo que deberías estudiar cómo funciona, cómo organizas eso" (EP2).

Por otro lado, los discursos de nuestros alumnos evidencian la necesidad de repensar la situación que en el plan de estudios tienen algunas materias. Parece que algunas asignaturas presentes en los últimos cursos aportan importantes claves para analizar y comprender la vida de las aulas. Esto nos invita a plantearnos si estos contenidos no deberían trabajarse en los cursos iniciales.

Atendiendo a ambas claves, podemos iniciar, acompañados por nuestros alumnos, un proceso de revisión sobre la estructura y organización de las materias que nos permita definir cuáles son las competencias, reflexiones y aprendizajes esenciales que deben vertebrar la formación inicial de los docentes.

\section{El papel de los tutores de escuela y las actividades desarrolladas en los centros}

En este apartado de análisis, reflexionamos sobre algunas prácticas educativas a las que acceden los alumnos en los centros y, especialmente, sobre las oportunidades que tienen para elaborar, junto a sus tutores, una visión crítica de las experiencias pedagógicas vividas.

Como bien sabemos, no todos los centros de prácticas y contextos escolares ofrecen las mismas oportunidades de aprendizaje a nuestros alumnos, como refleja el relato de esta alumna. 
[La primera vez que fui de prácticas] Lo que me encontré, mucha ficha, ehhh... 'niño siéntate, niño no abras la boca' $[. .$.$] Y también que decían que tra-$ bajaban con proyectos, pero hacían fichas, fichas, fichas. [...] En el segundo vi bastante diferencia porque son colegios distintos, uno era público y el otro era concertad (EI2).

Comprobamos cómo, en ocasiones, su estancia en el centro transcurre ejecutando tareas de aprendizaje amparadas bajo un modelo de enseñanza que prioriza la fragmentación del conocimiento y las actividades definidas por las editoriales. En otros casos, los centros y/o docentes proporcionan la oportunidad de participar en experiencias más innovadoras de enseñanza y que son más favorablemente valoradas por el alumnado de prácticas.

De igual forma, encontramos divergencias en cuanto al tipo de actividades fuera del marco de aula a las que les está permitido acceder. Es habitual que los alumnos tengan la posibilidad de observar el trabajo que realizan otros profesionales diferentes a su tutor y también de comprender el resto de actividades (de coordinación, reuniones con los padres...), más allá de tareas instructivas, que competen al maestro y que formarán parte de sus tareas y funciones como docentes. "He visto cómo trabajaba la orientadora, he visto cómo trabajaba también la PT y la AL de centro, la de religión, inglés" (EI3). "Reuniones de coordinación... he visto todas" (EP3).

Sin embargo, no siempre es posible pues cuestiones como la confidencialidad de la información o la reticencia de algunos maestros a exponer su trabajo pueden paralizar estas oportunidades de aprendizaje. "Nos dejaban al margen, no nos dejaban ir a las reuniones ni con los profesores ni con la PT ni nada. Al final es otra función más que vas que tener que desempeñar" (EI2).

Conscientes de la importancia de estos momentos pedagógicos, los alumnos demandan la creación de oportunidades para acudir a estos espacios de diálogo y de toma de decisiones que los maestros disponen en la escuela.

Finalmente, encontramos tutores, los menos, que optan por relegar al estudiante a un papel de observador: "el tutor me dijo que no podía hacer nada. Que tenía que estar sentada en una silla mirando cómo los niños hacían las cosas" (EP1), mientras la mayoría prefiere incorporarlo a la acción desde el principio y desde una posición mucho más horizontal: "he estado como una profesora más, he preparado clases, corregido exámenes" (EI3). Los alumnos interpretan que estas diferencias se vinculan a la titularidad del centro, a las características del tutor y al modelo educativo que promueven, desligándolo del periodo de prácticas en el que se encuentran.

Recordemos, en tal sentido, que la consigna desde la facultad es reservar un momento, al inicio de las prácticas, para observar la actividad del aula, a fin de favorecer la comprensión de la estructura y características de las dinámicas relacionales y de trabajo. Se considera esta reflexión fundamental para iniciar posteriormente la acción. Sin embargo, hemos comprobado que este momento de observación inicial no suele considerarse, por los tutores de los centros, como un tiempo con identidad propia y se convierte, más bien, en un eje transversal a lo largo de su estancia en el centro. Sin lugar a dudas, observar y actuar se configuran como herramientas esenciales para favorecer el desarrollo de docentes reflexivos y deliberativos en torno a su práctica. Sin embargo, esto requiere, por un lado, disponer de algunas claves organizativas y didácticas para comprender y significar lo que ocurre en un aula y, por el otro, cierta formación sobre cómo llevar a cabo una observación-participante. Probablemente, éste sea uno de los retos futuros en el practicum.

Además, para el desarrollo de estas claves de interpretación el establecimiento de espacios de diálogo y de reflexión conjunta con el tutor del centro se presenta como condición irrenunciable. Un diálogo compartido permitirá desvelar la complejidad 
de muchas situaciones educativas y comprender, críticamente, las acciones llevadas a cabo y aquellas otras que podrían haberse desarrollado alternativamente. Sin embargo, encontramos que son escasos los momentos de análisis y reflexión que el estudiantado desarrolla con sus tutores: "Yo creo que no están tanto a explicarte como a otras cosas: a que hagas, a que veas, a que te equivoques... Están más a hacer que a explicar. A ellos les parece simplemente lógico, no lo piensan" (EP1).

Incidir en la necesidad de que los tutores de los centros dediquen espacios y tiempos a interpretar junto con el alumnado el sentido de sus acciones y decisiones se perfila como otra línea de mejora ineludible del practicum. Además, esta reflexión compartida no sólo enriquecerá al alumnado. También los docentes podrán revisar y analizar su práctica fuera de la inmediatez y dirigir la mirada hacia lo importante, desplazando aquellos asuntos urgentes (y muchas veces irrelevantes) que copan diariamente la atención a un necesario segundo plano.

\section{El Portfolio: ¿̇herramienta de reflexión o documento-resumen?}

Durante su estancia en los centros el alumnado debe elaborar un portfolio en el que reflexionar y analizar la experiencia vivida. Este documento, que servirá, en última instancia, como evidencia de evaluación para el supervisor universitario, recoge dos tareas diferenciadas.

\section{El diario de prácticas}

El diario pretende constituir un documento reflexivo resultado de los procesos de observación de la actividad educativa. Su orientación no es la mera descripción, sino una labor más compleja de reconstrucción de la experiencia vivida (Zabalza, 2004) en la que debe incorporarse información de diversa naturaleza: documentación de las experiencias, interpretación de la actuación del docente, análisis de las acciones e interacciones del alumnado, relación con algunos aspectos teóricos, etcétera. Situar al alumnado ante este reto supone favorecer su desarrollo como futuros docentes reflexivos, incorporando a su acervo profesional estrategias de análisis y mejora educativa.

Algunos participantes destacan el diario como una de las experiencias más enriquecedoras del practicum. Escribiendo sus pensamientos y vivencias, los estudiantes pueden recodificar situaciones y observarse a sí mismos desde una óptica diferente, lo que posibilita la racionalización y comprensión de las prácticas y su transformación en fenómenos modificables (Zabalza, 2004). "El diario me parece bien para veas lo que tú has hecho. Igual tu actúas y no te paras a pensar en las cosas que vas haciendo durante el día $[. .$.$] a la hora de reflexionar te das$ cuenta de que debes cambiar o continuar por ese camino" (EIl).

Sin embargo, también apuntan algunos aspectos que obstaculizan su desarrollo:

El proceso de recogida de la información. La elaboración del diario está sustentada en los procesos de observación que los alumnos realizan durante su estancia en el centro. La observación supone, por un lado, educar la mirada y, por el otro, llevar a cabo una recogida de información sistemática y orientada. A lo largo del día, los estudiantes son testigos de múltiples acciones, tomas de decisiones, interacciones, conflictos, etcétera, pero en el diario deben reflejar únicamente la información relevante. Sin embargo, nuestros alumnos señalan que, durante su formación, no han adquirido las estrategias necesarias para llevar a cabo este proceso de atención dirigida.

Cuando lo estás haciendo dices, 'qué tontería más grande es esto'. Al final te das cuenta que acabas poniendo: 'este día hice esto, al día siguiente hice lo mismo, y al día siguiente lo mismo'. Hay muchas cosas que se repiten y al final acabas poniendo lo mismo o muy parecido. Hay poca selección (EI2). 
Además, los alumnos señalan dificultades en el momento de tomar las notas de campo para su diario. Este proceso difiere entre ellos: algunos optan por recoger todo lo sucedido en el aula, independientemente de su relevancia, para proceder, en un momento posterior, a seleccionar la información. Por otro lado, hay alumnos que realizan dicho discernimiento durante el propio acto de observar. "Lo más difícil es el diario [...] Puedes ir con una libreta pero al final no estás apuntando todo lo que pasa. Y llegas a casa, apuntas un día sí y otro día no...entonces llega un momento que no te acuerdas lo que ha pasado (EP3).

Diferencias en su elaboración. Si bien todos los alumnos comparten una guía común para el desarrollo del diario, existe gran divergencia en las indicaciones que aportan los diferentes supervisores de la facultad. Dichas disimilitudes se traducen en diarios y portfolios de una magnitud, características y profundidad muy diferentes, así como también en una exigencia y calificaciones muy dispares.

El problema es que unos tutores te dicen que lo hagas de una manera, otros que de otra e incluso algunos tutores te dicen que pongas solo 10 días, 10 que son los más importantes y ya está. [...] El primer tutor nos obligó a relacionar todo lo que poníamos en el diario, y lo que poníamos en el informe, con lo que habíamos visto en clase, todo lo que no estuviera relacionado no le interesaba. En el segundo caso fue más, pues, lo que te parecía importante lo ponías, lo que podías relacionar, lo relacionabas, fue un poco más libre (EIl).

Estas diferencias generan altas dosis de incertidumbre en el alumnado, especialmente en los momentos iniciales, que pueden verse atenuadas, como señala Rodríguez (2002), si los alumnos comparten la experiencia con los tutores de los centros y de la facultad. Planificar momentos de reflexión conjunta, con ambas figuras, es fundamental para guiar las tareas reflexivas del alumnado y favorecer procesos de análisis que sean verdaderamente críticos y reflexivos.

\section{El informe de prácticas}

En este informe los alumnos deben recoger reflexiones relativas a la organización y gestión del centro y a las implicaciones que tiene en el desarrollo de la vida de las aulas: el contexto, los diferentes agentes, la dirección del centro o los documentos de centro.

Algunos alumnos encuentran que la elaboración de este documento les aporta una visión más compleja de la vida escolar, ayudándoles a comprender cómo los aspectos y decisiones de centro condicionan la práctica de aula. "La parte del informe, a ver, está bien porque te obliga a mirar la documentación del centro y ver si está acorde la teoría con la práctica, te adentras en la mirada de la escuela, está bien que no te quedes sólo con lo que se hace en tu aula" (EI1).

Sin embargo, la mayoría de los estudiantes participantes no conciben una utilidad en la elaboración de este informe: "[Lo más difícil resultó] redactar el informe porque me aburre completamente. El diario te pones y lo haces. Pero el informe lo veo como mera formalidad y yo creo que el que lo lee también" (EP2).

Ésta y otras intervenciones, sobre las que nos iremos deteniendo, manifiestan cómo el informe de prácticas es concebido, en muchos casos, como un trabajo de trámite que les permite aprobar una asignatura cuyo mayor valor se sitúa en la "práctica" que tiene lugar en el corazón de las escuelas. Precisamente por la falta de sentido que encuentran es considerado como un trabajo tedioso y repetitivo que implica realizar prácticamente, en las tres asignaturas de Practicum, las mismas tareas (por ejemplo, el análisis relativo a los documentos de centro) o trabajos que se solapan con lo requerido en otras asignaturas (realización de unidades didácticas). "Es que tal y como está ahora sobra, es meter información por meter información. Es que no me parece útil que tú busques en la 
información del centro cuántas clases hay de infantil, cuántas de primaria, cuántos niños tienen beca del comedor... Y qué le aporta al tutor" (EP1).

A pesar de esta valoración que prevalece en sus discursos, consideramos necesario que los alumnos comprendan que "el centro escolar considerado como organización constituye un contexto clave para el desarrollo del currículum, el aprendizaje de los alumnos y la actividad docente que realizan profesores y profesoras" (González, 2009: 5). Sin embargo, es posible que el diseño del informe, con una estructura excesivamente rígida y tareas que se repiten con pocas alteraciones en las tres asignaturas de Practicum, no sea el espacio propicio para facilitar la comprensión de las dimensiones organizativas de la escuela ni la reflexión sobre los aspectos sociopolíticos que condicionan la enseñanza. Un informe más vivo, libre y personal se presenta como una opción considerada por algunos alumnos para mejorar este aspecto del portfolio:

Yo lo haría más sin tener que seguir un guion tan rígido. Me parece importante hablar del contexto, del tipo de alumnado... No tiene nada que ver la ubicación de unos centros con la de otros... eso condiciona lo que hay en las clases. Y eso me parece que es muy importante. Pero eso lo metería como una introducción al diario. Yo haría un súper diario. Pondría cómo es el centro, cómo está organizado... y luego ya me metería en el diario (EP3).

La función del supervisor de la universidad es también fundamental para detectar estas reflexiones que suponen una visión sobre la educación simplificada y ajena a sus condicionantes. A continuación, reflexionamos sobre su papel.

\section{Los supervisores de la universidad: de evaluadores a amigos-críticos}

Hemos visto que las experiencias de prácticas a las que el alumnado accede son muy disímiles y no en todos los casos ofrecen las mismas oportunidades de participar y aprender. Independientemente de la calidad educativa de las mismas, pueden obtenerse aprendizajes muy relevantes promoviendo procesos de análisis y de reflexión crítica sobre lo que se ha visto y realizado. El papel del supervisor de practicum en cuanto a incitador de esos procesos se constituye como fundamental. A pesar de su importancia, colegimos de los discursos de nuestros participantes que no todos los modos de ejercer este rol se corresponden con la necesidad perfilada.

Encontramos, de este modo, un número elevado de tutores que limitan su acción a la explicación de la estructura del portfolio y a responder a las posibles dudas individuales que, sobre su elaboración, pudieran surgir: "Sí, pero vamos, de que te pregunten que cómo vas y ya está. Y dudas y ya" (EI3). Su papel queda relegado, así, al de meros evaluadores de un trabajo del que no se ofrece ningún feedback ni orientación durante el proceso. Quizá éste sea uno de los motivos que explican por qué los alumnos viven la elaboración del informe como un trámite sustentado en la repetición, en lugar de concebirlo como un espacio de reflexión y de aprendizaje.

No obstante, reconocemos otro perfil de supervisor comprometido con la elaboración del portfolio como herramienta de reflexión y análisis que perseguimos, lo que normalmente se materializa a través de seminarios grupales (entre los alumnos, que tutoriza el mismo supervisor). De este modo, los tutores desempeñan el papel de "amigos críticos" que trasladan a los alumnos preguntas en clave de análisis para que profundicen y amplíen su reflexión. Al mismo tiempo, los compañeros de otras aulas o centros se convierten en interrogadores sobre las prácticas vividas, y estos encuentros son espacios para complementar la mirada crítica que los alumnos desarrollan sobre sus prácticas. En conclusión, los seminarios bajo este perfil de supervisor se orientan a la generación de espacios dialógicos que facilitan la articulación de la teoría y la práctica, así como el análisis y la reflexión 
sobre las experiencias idiosincrásicas que tienen los alumnos.

\section{Conclusiones}

En los últimos años, el sistema universitario español se ha embarcado en un complejo proceso de cambio hacia el EEES. La institución universitaria se halla en una situación complicada e imbuida de intereses de muy diversa índole que combina, con tirantez, grandes oportunidades y considerables riesgos. Con todo ello, la convergencia se presenta como la ocasión para repensar la enseñanza dentro de una institución universitaria que "tiende a ir lastrada con métodos que tienen una continuidad y una resistencia al cambio infalibles" (Brockbank y McGill, 2002: 109).

En este artículo hemos presentado algunas reflexiones sobre el practicum en este marco contextual que realizan los participantes de una investigación desarrollada en la Universidad de Cantabria. En general, observamos que la ampliación del periodo que los alumnos desarrollan en las escuelas se manifiesta como el único cambio palpable dentro del practicum, pues aspectos como la función del supervisor universitario, del tutor-maestro o la estructura del portfolio permanecen prácticamente inalterables.

Así, en nuestros alumnos participantes pervive una tendencia a divorciar los constructos de teoría y práctica y a valorar este segundo en detrimento del otro. Uno de los peligros de esta concepción es que conduce a interpretar cualquier experiencia como valiosa y, en consecuencia, al practicum como periodo beneficioso en sí mismo. Esto puede acarrear una "tendencia a imitar y reproducir el comportamiento social y profesional que contempla como mayoritario y aparentemente exitoso en el medio social del aula y la escuela" (Pérez Gómez, 1999: 639), sin reflexionarlo y analizarlo de manera crítica.

La función del supervisor universitario ante esta realidad es fundamental y definir sus tareas en cuanto a acompañante y orientador de los procesos reflexivos del alumnado se manifiesta como una línea de acción prioritaria. Sin embargo, observamos una heterogeneidad muy grande en las prácticas de los diferentes supervisores, encontrando que muchas de ellas no se ajustan al perfil requerido. Replantear el rol del supervisor en este sentido y establecer espacios de acuerdo que permitan homogeneizar actuaciones es fundamental para avanzar mejoras en el practicum.

Al mismo tiempo, constatamos que la figura del tutor del centro protagoniza fundamentalmente momentos de acción y una enseñanza sustentada en el modelado. Son más escasos, sin embargo, los espacios distanciados del quehacer diario destinados a analizar, de manera conjunta con el estudiante, momentos relevantes de la práctica desarrollada.

En este sentido, resaltamos la necesidad de establecer una relación más estrecha entre la institución escolar y la universitaria y, consecuentemente, de proporcionar un partenariado compartido y sinérgico (Allen et al., 2013; Zabalza, 2007). Una coordinación fluida y una coherencia en las actuaciones son fundamentales para proporcionar experiencias de valor formativo (Van Velzen et al., 2012) y generar los espacios dialógicos insoslayables que deben plantearse para reflexionar colaborativamente sobre la experiencia de prácticas. Desgraciadamente, los tutores de la universidad y de la escuela suelen desconocer el trabajo que cada uno realiza en la supervisión del practicum. Además de una tensión formativa, la incoherencia entre ambas instituciones puede también traducirse en una incertidumbre en el alumno de no saber a quién responder (Allen, 2011; Correa, 2011). En tal línea, Zeichner (2010) propone la generación de "espacios híbridos", es decir, lugares de formación del profesorado en los que converjan, en un plano de horizontalidad, los conocimientos generados en la academia y en el entorno escolar, permitiendo al alumnado beneficiarse de diversas fuentes de experiencia.

Finalmente, los estudiantes llaman la atención sobre la necesidad de modificar algunos aspectos del 
portfolio poco relevantes para el aprendizaje y convertirlo en un espacio más personal de análisis de la práctica desarrollada. En este aspecto, las tecnologías, en sintonía con sus intereses y su modo de interacción actual, brindan un espacio de posibilidades para este fin. Contamos ya con algunas experiencias que hacen uso de las TIC, como los e-portfolios

\section{Referencias}

Allen, Jeanne (2011), “Stakeholders' perspectives of the nature and role of assessment during practicum", en Teaching and Teacher Education, núm. 27, pp. 742-750.

Allen, Jeanne, Angelina Ambrosetti y David Turner (2013), "How school and university supervising staff perceive the pre-service teacher education practicum: a comparative study", en Australian Fournal of Teacher Education, vol. 38, núm. 4, pp. 108-128.

Aneas, Assumpta, Nuria Rajadell, Angels Renom e Inmaculada Armadans (2013), "Practicum: transición, transdisciplina y transformación”, en Un Practicum para la formación integral de los estudiantes, Santiago de Compostela, Andavira, pp. 149-158.

Angrosino, Michael (2012), Etnografia y observación participante en investigación cualitativa, Madrid, Morata.

Beck, Clive y Clare Kosnik (2002), "Associate teachers in preservice education: clarifying and enhancing their role", en Fournal of Education for Teaching, vol. 26, núm. 3, 207-224.

Brockbank, Anne e Ian Mcgill (2002), Aprendizaje reflexivo en la educación superior, Madrid, Morata.

Cebrián de la Serna, Manuel (2010), "Supervisión con e-portfolios y su impacto en las reflexiones de los estudiantes en el practicum. Estudio de caso", en Revista de Educación, núm. 354, pp. 183-208.
(Cebrián de la Serna, 2010) o los weblogs portfolios (Chuang, 2010; Portillo y Cano, 2015), que mejoran la comunicación tutor universitario-alumno, ofrecen la posibilidad de retroalimentar reflexiones de un modo mucho más continuo y facilitan la compartición de experiencias y recursos con otros estudiantes.
Chuang, Han (2010), "Weblog-based electronic portfolios for student teachers in Taiwan”, en Educational Technology Research and Development, núm. 58, pp. 211-227.

Colás, Pilar (1998), Investigación Educativa, Sevilla, Alfar.

Correa, Enrique (2015), "La alternancia en la formación inicial docente: vía de profesionalización", en Educar, vol. 51, núm. 2, pp. 259-275.

Correa, Enrique (2011), "La práctica docente: una oportunidad de desarrollo profesional", en Perspectiva Educacional, núm. 50, vol. 2, pp. 77-95.

Clarke, Anthony, Valerie Triggs y Wendy Nielsen (2014), "Cooperating teacher participation in teacher education: a review of the literature", en Review of Educational Research, vol. 84, núm. 2, pp. 163-202.

Denzin, Norman e Ivonna Lincoln (2012), Manual de investigación cualitativa, Barcelona, Gedisa.

Díaz de Rada, Ángel (2007), Etnografía y técnicas de investigación antropológica, Madrid, UNED.

Ferrier-Kerr, Jenny (2009), "Establishing professional relationships in practicum settings", en Teaching and Teacher Education, núm. 25, pp. 790-797.

Flick, Uwe (2009), Introducción a la investigación cualitativa, Madrid, Morata.

González, María-Teresa (2009), "Las organizaciones escolares: dimensiones y características”, en Organización 
y gestión de centros escolares: dimensiones y procesos, Madrid, Pearson Educación, pp. 25-40.

González-Sanmamed, Mercedes (2001), “¿Qué se aprende en el practicum? ¿Qué hemos aprendido en el practicum?", en Actas del VI Simposium de Poio.

González-Sanmamed, Mercedes y Eduardo Fuentes (2011), "El Practicum en el aprendizaje de la profesión de docente", en Revista de Educación, núm. 354, pp. 47-70.

Hammersley, Martyn y Paul Atkinson (2003), Etnografia: métodos de investigación, Barcelona, Paidós.

Huber, Günter (2003), "Introducción al análisis cualitativo de datos", en Metodología para la realización de proyectos de investigación y tesis doctorales, Madrid, Universitas, pp. 81 129.

Imbernón, Francisco y María Teresa Colén (2015), "Los vaivenes de la formación inicial del profesorado. Una reforma siempre inacabada”, en Tendencias Pedagógicas, núm. 25, pp. 57-76.

Korthagen, Fred, John Loughran y Tom Russell (2006), "Developing fundamental principles for teacher education programs and practices", en Teaching and Teacher Education, núm. 22, pp. 1020-1041.

Kvale, Steinar (2011), Las entrevistas en investigación cualitativa, Madrid, Morata.

Martínez Bonafé, Jaume (2013), "La reformas en la formación inicial del profesorado. ¿Pero cuáles son los buenos saberes de las buenas maestras?", en Revista Interuniversitaria de Formación del Profesorado, vol. 78, núm. 3, pp. 89-102.

Melek, Ebru (2012), "Idiographic roles of cooperating teachers as mentors in pre-service distance teacher education", en Teaching and Teacher Education, núm. 28, pp. $818-826$.

Mena, Juanjo, Marisa García, Anthony Clarke y Anastasio Barkatsas (2015), "An analysis of three different approaches to student teacher mentoring and their impact on knowledge generation in practicum settings", en European Fournal of Teacher Education, vol. 39, núm. 1, pp. 53-76.

Patrick, Rachel (2013), "Don't rock the boat: conflicting mentor and pre-service teacher narratives of professional experience", en The Australian Educational Researcher, núm. 40, pp. 207-226.

Pérez Gómez, Ángel (1999), "El practicum de enseñanza y la socialización profesional de los futuros docentes", en Desarrollo profesional del docente: politica, investigación y práctica, Madrid, Akal, pp. 636-660.

Portillo, María y Elena Cano (2015), "Regulating the writing process in the teacher training practicum guided by feedback at the University of Barcelona”, en Cambridge Fournal of Education, <DOI:http://dx.doi.org /10.1080/0305764X.2015.1011083>.

Rapley, Tim (2014), Los análisis de la conversación, del discurso $y$ de documentos en investigación cualitativa, Madrid, Ediciones Morata.

Rodríguez, Ana (2002), Cómo innovar en el Prácticum de Magisterio. Aplicación del portfolios a la enseñanza universitaria, Oviedo, Septem.

Rodríguez-Hoyos, Carlos, Adelina Calvo e Ignacio Haya (2015), "La tutoría académica en la educación superior. Una investigación a partir de entrevistas y grupos de discusión en la Universidad de Cantabria (España)", en Revista Complutense de Educación, vol. 26, núm. 2, pp. 467-481.

Smith, Kari (2010), "Assessing the practicum in teacher education. Do we want candidates and mentors to agree?", en Studies in educational evaluation, núm. 36, pp. 36-41.

Sorensen, Peter (2014), "Collaboration, dialogue and expansive learning: the use of paired and multiple placements in the school practicum", en Teaching and Teacher Education, núm. 44, pp. 128-137.

Tójar, Juan Carlos (2006), Investigación cualitativa: comprender y actuar, Madrid, La Muralla.

Valle, Javier y Jesús Manso (2010), "La nueva formación inicial del profesorado de educación secundaria: modelo para la selección de buenos centros de prácticas", en Revista de Educación, núm. 354, pp. 267-290.

Van Velzen, Corinne, Monique Volman, Mieke Brekelmans y Simone White (2012), "Guided workbased learning: sharing practical teaching knowledge with student teachers", en Teaching and Teacher Education, 
núm. 28, pp. 229-239.

Ventura, Javier (2005), "El practicum en los estudios pedagógicos y la inserción laboral. Nuevos enfoques ante el reto europeo", Tesis doctoral inédita, Departamento de Teoría e Historia de la Educación, Barcelona, Universidad de Barcelona.

Wilson, Gary y John I'Anson (2006), "Reframing the practicum: constructing performative space in inicial teacher education", en Teaching and Teacher Education, núm. 22, pp. 353-361.

Zabalza, Miguel Ángel (2013), "El practicum como contexto de aprendizaje”, en P. C. Muñoz, M. Raposo, M. González, M. E. Martínez, M. Zabalza y A. Pérez (eds.), Un practicum para la formación integral de los estudiantes, Santiago de Compostela, Andavira, pp. 51-68.

Zabalza, Miguel Ángel (2011), "El practicum en la formación universitaria: estado de la cuestión", en Revista de Educación, núm. 354, pp. 21-43.

Zabalza, Miguel Ángel (2007), "Buenas prácticas en el Practicum: bases para su identificación y análisis”, en
Actas del IX Simposium de Poio, recuperado de: http:// redaberta.usc.es/uvi/public_html/html/actas2007. htm.

Zabalza, Miguel Ángel (2006), "La convergencia como oportunidad para mejorar la docencia universitaria", en Revista Interuniversitaria de Formación del Profesorado, vol. 20, núm. 3, pp. 37-69.

Zabalza, Miguel Ángel (2004), Diarios de clase. Un instrumento de investigación y desarrollo profesional, Madrid, Narcea.

Zeichner, Kenneth (2002), "Beyond traditional structures of student teaching", en Teacher Education Quarterly, vol. 29, núm. 2, pp. 59-64.

Zeichner, Kenneth (2005), "Becoming a teacher educator: a personal perspective", en Teaching and Teacher Education, núm. 21, pp. 117-124.

Zeichner, Kenneth (2010), "New epistemologies in teacher education. Rethinking the connections between campus courses and practical experiences in Teacher Education at university", en Fournal of Teacher Education, núm. 61, pp. 89-99.

Cómo citar este artículo:

Saiz-Linares, Ángela y Noelia Ceballos-López (2019), “El practicum de magisterio a examen: reflexiones de un grupo de estudiantes de la Universidad de Cantabria", en Revista Iberoamericana de Educación Superior (RIES), México, UNAM-IISUE/Universia, vol. X, Núm. 27, pp. 136-150, DOI: dx.doi.org/10.22201/iisue.20072872e.2019.27.344 [consulta: fecha de última consulta]. 